\title{
VARIEDADES TRADICIONALES DE TOMATE DE LA COMUNIDAD VALENCIANA DEL BANCO DE GERMOPLASMA DEL COMAV
}

\author{
M. J. Díez ${ }^{* 1}$, J.V. Valcárcel ${ }^{1}$, J. Torres ${ }^{1}$, \\ A. Granell ${ }^{2}$, J. Prohens ${ }^{1}$, S. Soler ${ }^{1}$ \\ ${ }^{1}$ Instituto de Conservación y Mejora de la Agrodiversidad Valenciana \\ (COMAV). Universitat Politècnica de València. Camino de Vera, s/n, \\ 46022 Valencia, España. \\ ${ }^{2}$ Instituto de Biología Molecular y Celular de Plantas Primo Yufera. \\ Consejo Superior de Investigaciones Científicas- Universitat Politècnica de \\ València. Camino de Vera, s/n, 46022 Valencia, España.

\section{*mdiezni@,btc.upv.es}

\begin{abstract}
The genebank of COMAV holds a collection of about 3000 accessions of tomato. More than 600 come from the Comunidad Valenciana. Three hundred and fifteen accessions come from the Valencia province, 153 from Alicante and 164 from Castellón. Characterization data of a large part of the collection are available. One hundred and thirty two belong to the 'Valenciano' type and were collected in expeditions carried out from the beginning of the eighties until the present. A characterization assay of 51 accessions of 'Valenciano' type was conducted in El Perelló in the spring-summer season. This allowed their grouping in four groups according to their fruit morphology These grups were: "Masclet", with heart shaped fruits pointed at the stilar area typical of this variety, "Femella" with wider and less pointed fruits, "Xato" with flattened and ribbed fruits, and "Rosada" with pink coloured fruits. A high variability was found for all descriptors recorded, except for growth type, being almost all accessions of the indeterminate type. The fruit weight ranged from $201 \mathrm{~g}$ in the "Rosada" type to $362 \mathrm{~g}$ in the "Xato" group. The less productive grup was the "Xato" one with moderate yields. In this case the bigger fruit size gave rise to a more irregular fruit set and consequently to a lower yield. The highest percentage of accessions with high yield was found in the "Femella" type with a $37 \%$ of accessions scored as 7 , being 9 the maximum score for this
\end{abstract}


characteristic. The high variability found for all descriptors scored and the adaptation to a wide range of agroclimatic conditions ensure the value of this collection for being incorporated in breeding programmes or for the direct cultivation of the best accessions.

Keywords: germplasm, varietal types, morphologic variability

\section{Resumen}

El banco de germoplasma del COMAV conserva una colección de tomate de cerca de 3000 entradas. Más de 600 proceden de la Comunidad Valenciana. Ciento cincuenta y tres fueron recolectadas en la provincia de Alicante, 164 en Castellón y 315 en Valencia. Se dispone de datos de caracterización de una parte importante de las entradas. Ciento treinta y dos entradas pertenecen al tipo 'Valenciano' y fueron colectadas en expediciones de recolección realizadas desde principios de los años 80 hasta el presente. Cincuenta y una entradas del tipo 'Valenciano' han sido caracterizadas recientemente en El Perelló en cultivo de primavera-verano. De acuerdo a los datos referentes a la morfologia de fruto se han separado en cuatro grupos: "Masclet", con frutos en forma de corazón y con el extremo estilar apuntado, característica propia de esta variedad, "Femella" con frutos más anchos y menos apuntados, "Xato" con frutos más achatados y acostillados, y "Rosada" con frutos de color rosa. Se encontró una elevada variabilidad para todos los descriptores tomados, excepto para tipo de crecimiento, siendo prácticamente todas las entradas de tipo indeterminado. El peso del fruto varió entre los $201 \mathrm{~g}$ del tipo "Rosada" y los $362 \mathrm{~g}$ en el grupo "Xato". El grupo menos productivo fue el "Xato" con una producción moderada. En este caso, el elevado peso del fruto ocasiona un cuajado más irregular y consecuentemente se produce una disminución de la producción. El porcentaje más elevado de entradas con producción alta se encontró en el tipo "Femella", con un $37 \%$ de las mismas con puntuación 7 , siendo la puntuación máxima 9 para esta característica. La gran variabilidad encontrada para la mayoría de caracteres controlados y la adaptación a un amplio rango de condiciones agroclimáticas pone en valor esta colección para ser utilizada en programas de mejora o para cultivar directamente las mejores entradas.

Palabras clave: germoplasma, tipos varietales, variabilidad morfològica 


\section{1.- INTRODUCCIÓN}

El Banco de Germoplasma del COMAV empezó las recolecciones de variedades tradicionales de cultivos hortícolas a principios de los años 80, cuando era cada vez más patente la erosión genética que estaba reduciendo la base genética de los cultivos (Cebolla-Cornejo et al., 2007) . En la actualidad mantiene una colección activa de cerca de 14.000 entradas. Las semillas se conservan ex situ desecadas y a una temperatura de aproximadamente $4{ }^{\circ} \mathrm{C}$. Estas condiciones garantizan la viabilidad de la semilla durante decenas de años (Rao et al., 2006) y permiten el mantenimiento de grandes colecciones de variedades que no podrían ser mantenidas in situ por los agricultores debido a su gran tamaño. El banco de germoplasma participa en la conservación de estos materiales, manteniéndolos en óptimas condiciones y haciéndolos accesibles a personas o entidades interesadas como agricultores, centros de investigación públicos y privados, administración pública, etc. El número de entradas suministradas por el Banco de Germoplasma del COMAV en los últimos 5 años asciende a más de 13.000.

La colección de tomate de variedades tradicionales españolas es de algo más de 3000 entradas, de las cuales 632 proceden de la Comunidad Valenciana. Las expediciones de recolección se hicieron en unos casos recopilando previamente información acerca de la existencia de variedades tradicionales de reconocido renombre, y en otros recolectando directamente en lugares de los que no se disponía de información previa. Esto nos ha permitido disponer de entradas de todas las comarcas de la Comunidad. Durante las colectas se siguieron las recomendaciones del International Plant Genetic Resources Institute (IPGRI, 1996), rellenando los datos de pasaporte recomendados relativos al origen de las muestras, características del suelo y clima, uso y valor específico de cada entrada, etc. Toda esta información está accessible para los usuarios interesados en adquirir y cultivar variedades tradicionales.

\section{2.- ORIGEN Y TIPOS VARIETALES DE TOMATE DE LA COMUNIDAD VALENCIANA CONSERVADOS EN EL BANCO DE GERMOPLASMA}

El número de entradas colectadas en la Comunidad Valenciana es de 632, procediendo 153 de la provincia de Alicante, 164 de Castellón y 315 de Valencia y estando repesentadas 33 comarcas de la Comunidad. De algunas entradas se desconoce su comarca de origen (Tabla 1). 
Tabla 1.- Procedencia de las entradas de tomate conservadas en el banco de germoplasma del COMAV

\begin{tabular}{|c|c|c|c|}
\hline Provincia & Comarca & $\begin{array}{c}\text { Número de } \\
\text { entradas }\end{array}$ & $\begin{array}{l}N^{0} \text { entradas de } \\
\text { tipo Valenciano }\end{array}$ \\
\hline \multirow{9}{*}{ Alicante } & Alacantí & 32 & 1 \\
\hline & Alcoià & 5 & 1 \\
\hline & Alt Vinalopó & 28 & 3 \\
\hline & Baix Segura & 19 & \\
\hline & El Comtat & 18 & 1 \\
\hline & Marina Alta & 28 & \\
\hline & Marina Baixa & 3 & \\
\hline & Vinalopó & 7 & \\
\hline & Vinalopó Mitjà & 13 & 1 \\
\hline \multirow{8}{*}{ Castellón } & Alcalaten & 12 & \\
\hline & Alt Maestrat & 12 & \\
\hline & Alt Millars & 26 & 1 \\
\hline & Alt Palancia & 28 & 9 \\
\hline & Baix Maestrat & 24 & \\
\hline & Els Ports & 16 & \\
\hline & Plana Alta & 23 & \\
\hline & Plana Baixa & 23 & 4 \\
\hline \multirow{17}{*}{ Valencia } & Camp de Morvedre & 9 & 5 \\
\hline & Camp de Túria & 19 & 9 \\
\hline & Canal de Navarres & 9 & 2 \\
\hline & Costera & 3 & 1 \\
\hline & Foia de Bunyol & 19 & 5 \\
\hline & Horta Nord & 76 & 34 \\
\hline & Horta Oest & 11 & 5 \\
\hline & Horta Sud & 11 & 4 \\
\hline & La Safor & 11 & 6 \\
\hline & Requena-Utiel & 12 & 2 \\
\hline & Ribera Alta & 29 & 12 \\
\hline & Ribera Baixa & 20 & 12 \\
\hline & Racó d'Ademús & 34 & 1 \\
\hline & Serrans & 16 & 3 \\
\hline & Vall d'Albaida & 22 & 5 \\
\hline & Vall de Confrents & 3 & \\
\hline & Comarca desconocida & 11 & 5 (Valencia) \\
\hline & $\overline{\mathbf{A L}}$ & 632 & 132 \\
\hline
\end{tabular}


Aproximadamente la mitad de la colección ha sido caracterizada y se conoce el tipo de fruto o la variedad a la que pertenece cada entrada. Del resto se dispone de datos de pasaporte, en los que a menudo se incluye información sobre el tipo de fruto o tipo varietal. En la Tabla 2 se indican los tipos a los que pertenecen las entradas conservadas, separando la información en dos columnas según la procedencia de la misma (caracterización en el banco de germoplasma o datos de pasaporte). Algunos tipos aluden a variedades tradicionales de reconocido prestigio ('Valenciano', 'Muchamiel', 'de Penjar', etc.) (Figás et al., 2015), mientras que otros se han denominado atendiendo a las características de los frutos (Achatado, Acorazonado, Amarillo, etc.).

Como puede verse, el tipo predominante es el 'Valenciano', con 132 entradas. Los tipos 'De Penjar', 'Muchamiel', 'de Conserva', 'Marmande' o 'del Pebre', también cuentan con un elevado número de entradas. Un grupo significativo de entradas no se encuadra en ninguno de estos tipos, en ocasiones porque no se tienen datos y en otras porque no se adaptan a ninguno de los tipos descritos, aunque son cultivadas y valoradas en sus lugares de procedencia. 
Tabla 2.- Entradas de la colección según las características de sus frutos, el tipo varietal y provincia de origen

\begin{tabular}{|c|c|c|c|c|c|c|c|}
\hline \multirow[b]{3}{*}{ Achatado } & \multicolumn{3}{|c|}{ Entradas caracterizadas } & \multicolumn{3}{|c|}{ Información de datos de pasaporte } & \multirow[b]{2}{*}{ TOTAL } \\
\hline & Alacant & Castelló & València & Alacant & Castelló & València & \\
\hline & & 2 & 4 & & & & 6 \\
\hline Achatado rosa & & 1 & 3 & & & & 4 \\
\hline Acorazonado & 1 & & & & & & 1 \\
\hline Amarillo & & & & & & & 3 \\
\hline Beef & & 2 & 1 & & & & 3 \\
\hline Beef-rosa & & & & & & & 2 \\
\hline Borseta & & & & & & & 1 \\
\hline Centenares & & & & & & 1 & 1 \\
\hline Cherry & & & 2 & 2 & & 3 & 7 \\
\hline Cocktail & & & 1 & & & & 3 \\
\hline Cocktail/alargado & & & & & & & 1 \\
\hline Conserva & & 10 & 8 & 7 & 2 & 3 & 36 \\
\hline Corazón de buey & 2 & & 1 & & & & 3 \\
\hline Corazon de buey rosa & 1 & & & & & & 1 \\
\hline Cuarenteno & 2 & & 4 & & & 4 & 10 \\
\hline De la pera & 1 & & 1 & & & & 2 \\
\hline De penjar & 6 & 28 & 8 & 4 & 14 & 9 & 69 \\
\hline De secar & & & & & & 1 & 1 \\
\hline Elchero & 2 & & & & & & 2 \\
\hline Flor de Balabre & 1 & & 1 & & & & 2 \\
\hline Gordo rojo & & 1 & 2 & 1 & 5 & 3 & 12 \\
\hline Gordo rosa & & 1 & 4 & & 1 & 3 & 9 \\
\hline Marmande & & 5 & 7 & & & 1 & 16 \\
\hline Marmande negro & & & 1 & & & & 1 \\
\hline Marmande rosa & & 6 & 5 & & & & 11 \\
\hline Monserrat & & & & & & & 2 \\
\hline Moruno & & & & 1 & 3 & 2 & 8 \\
\hline Muchamiel & & & 5 & 2 & 1 & 1 & 37 \\
\hline Negro & & & 2 & & 1 & & 4 \\
\hline Palo santo & & & & & 3 & & 3 \\
\hline Pimiento & & 4 & 10 & & & & 15 \\
\hline Redondo & & & 5 & & & & 7 \\
\hline Redondo rosa & & & 1 & & & & 1 \\
\hline Rosa & & & 4 & & 3 & 6 & 15 \\
\hline Tres cantos & 1 & & & & & 1 & 2 \\
\hline Valenciano & 5 & 10 & 81 & 2 & 3 & 30 & 130 \\
\hline Valenciano rosa & & 1 & & & & & 2 \\
\hline TOTAL & & 80 & 166 & 19 & 36 & 68 & 433 \\
\hline
\end{tabular}




\section{3.- LA COLECCIÓN DE TOMATE 'VALENCIANO' DEL COMAV}

Ciento treinta y dos entradas procedentes de las tres provincias y de 23 comarcas son de tipo 'Valenciano' (Tabla 1), uno de los más apreciados en la Comunidad Valenciana (Soler et al., 2016). Los materiales conservados en el banco de germoplasma son recolectados directamente de los agricultores por lo que no se trata de variedades uniformes, sino que existe cierta variación entre plantas de una misma entrada, lo que por otra parte representa una ventaja puesto que permite realizar programas de selección. Diversas causas pueden explicar esta variación como son la existencia de cruzamientos espontáneos entre distintas variedades, dada la exerción estigmática frecuente, o la mezcla de semillas (Cortés-Olmos et al., 2015). El tipo 'Valenciano' se caracteriza por presentar distintas morfologías de fruto, a veces incluso en la misma planta, los llamados "Rotllos", "Femella" y "Masclet" (Cebolla, 2005). Los "Rotllos" son tomates achatados y deformes, procedentes de flores fasciadas que suelen aparecer con mayor frecuencia en los primeros racimos. Los de tipo "Femella" son frutos entre ligeramente achatados y acorazonados, pero sin presentar de forma pronunciada el alargamiento de la zona estilar. Por último, el "Masclet" es el tipo de fruto más valorado, de forma acorazonada y con la región estilar alargada. Es la existencia de esta región pistilar más o menos apuntada (en frutos "Masclet" o "Femella" respectivamente) lo que caracteriza de forma general a este tipo de tomate tradicional de la Comunidad Valenciana. Dependiendo de cada selección, realizada por los agricultores, se presenta de forma predominante la forma "Femella" o "Masclet" lo que ocasiona, muchas veces, que los agricultores se refieran a sus selecciones con distintos nombres en función de la forma o formas de fruto predominante en las plantas. 
Para describir las características de las entradas se ha escogido un conjunto de 51 entradas de tipo 'Valenciano' que fueron cultivadas conjuntamente en El Perelló en el ciclo de cultivo primavera-verano, en invernadero de plástico, en suelo arenoso y aplicando las técnicas y tratamientos habituales de este cultivo. Este ensayo tuvo lugar en el marco del proyecto TRADITOM. La caracterización se realizó empleando los descriptores desarrollados por el IPGRI con modificaciones realizadas en función de la diversidad contenida en el conjunto de entradas caracterizadas en dicho proyecto (IPGRI, 1996). En función de las características de los frutos, las entradas se han agrupado en cuatro tipos, "Femella" (41 entradas), "Masclet" (4 entradas), "Xato" (alude a la forma achatada de los frutos, por lo general de gran tamaño, 4 entradas) y "Rosada" (alude al color del fruto, 2 entradas) (Figura 1).

Las características comunes al conjunto de entradas estudiadas son el crecimiendo indeterminado de las plantas, aunque en ocasiones ha habido entradas con plantas de crecimiento semideterminado, follaje de porte colgante que cubre bien los frutos evitando el asoleado o planchado, inflorescencias irregulares y compuestas, características de la variedad y por lo general con hojas o brotes, aunque para este carácater existe variabilidad. En cuanto a la flor, la posición del estilo con respecto a las anteras es variable, siendo muy frecuente que estén al mismo nivel o que sobresalgan del cono de anteras. Esto influye sobre el cuajado de los frutos y también sobre las precauciones a tomar para la regeneración de este tipo de entradas a fin de evitar polinizaciones cruzadas con otras entradas vecinas. La mayoría de frutos son de color rojo, excepto los del tipo "Rosada". Para el color de los hombros existe una marcada variabilidad en todos los grupos, excepto en el "Rosada" y "Masclet", que son de color verde medio y en el caso específico de los "Masclet" verde oscuro (Figura 1, L, LL, M, N).

La producción, evaluada cualitativamente con valores desde 1 (producción escasa) a 9 (producción muy elevada), fue mayor en las variedades de tipo "Rosada", que tuvieron los frutos de menor peso (media de $201 \mathrm{~g}$ ) pero un buen cuajado (Tabla 3). El tipo menos productivo fue el "Xato" con producciones moderadas o medias. Éste es el tipo con frutos de mayor peso (media de $362 \mathrm{~g}$ ) lo cual repercute en un cuajado más irregular. El mayor porcentaje de entradas muy productivas se encontró en el tipo 
"Femella" con un 37\% de entradas con una valoración de 7, siendo 9 el valor máximo. Hay que señalar que ninguna entrada alcazó este valor, que se correspondería aproximadamente con una producción de más de 5 $\mathrm{kg} /$ planta. Todas las entradas tuvieron frutos multiloculares, especialmente las de tipo "Femella" y "Xato", que llegaron en algunos casos a tener frutos con 19 y 25 lóculos respectivamente. Esto confiere a los frutos la carnosidad típica de esta variedad. La fasciación estuvo presente en todos los tipos, dando lugar a los frutos de tipo "rotllo", que a pesar de su excesivo tamaño, acostillado fuerte y mayor o menor deformidad, son también apreciados por su calidad, carnosidad y sabor. Las cicatrices peduncular y estilar (irregular en la gran mayoría de las entradas) no produjeron un elevado porcentaje de destrío, a pesar del gran tamaño de los frutos.

Tabla 3.- Valores medios, máximo y mínimo para peso del fruto, número de lóculos, Brix y producción de los cuatro grupos de entradas de tipo 'Valenciano'

\begin{tabular}{|c|c|c|c|c|c|c|c|c|}
\hline \multirow[b]{2}{*}{ Tipo } & \multicolumn{2}{|c|}{ Peso del fruto } & \multicolumn{2}{|c|}{ Número de lóculos } & \multicolumn{2}{|c|}{${ }^{o}$ Brix } & \multicolumn{2}{|r|}{ Producción } \\
\hline & Media & $\begin{array}{l}\text { Valores } \\
\text { mínimo y } \\
\text { máximo }\end{array}$ & Media & $\begin{array}{l}\text { Valores } \\
\text { mínimo y } \\
\text { máximo }\end{array}$ & Media & $\begin{array}{c}\text { Valores } \\
\text { mínimo } \\
\text { y } \\
\text { máximo }\end{array}$ & $\begin{array}{c}\text { Valor } \\
\text { medio } \\
1 \text { (escasa) } \\
\text { a } 9 \text { (alta) }\end{array}$ & $\begin{array}{l}\text { Puntuación } \\
\text { producción ( } \mathrm{n}^{\mathrm{o}} \\
\text { entradas) }\end{array}$ \\
\hline "Femella" & 269 & $141-463$ & 9.6 & $4,4-19,2$ & 3.62 & $2,9-4,1$ & 5,7 & $3(3), 5(17), 6(6), 7(15)$ \\
\hline "Masclet" & 275 & $203-448$ & 8.8 & $8,1-9,5$ & 3.38 & $3,1-3,7$ & 5,5 & $5(3), 7(1)$ \\
\hline "Xato" & 362 & $212-645$ & 13.4 & $8,2-25,0$ & 3.72 & $3,2-4,1$ & 4,5 & $3(1), 5(3)$ \\
\hline "Rosada" & 201 & $145-257$ & 7.3 & $6,1-8,4$ & 3.54 & $3,4-3,6$ & 6,5 & $6(1), 7(1)$ \\
\hline
\end{tabular}




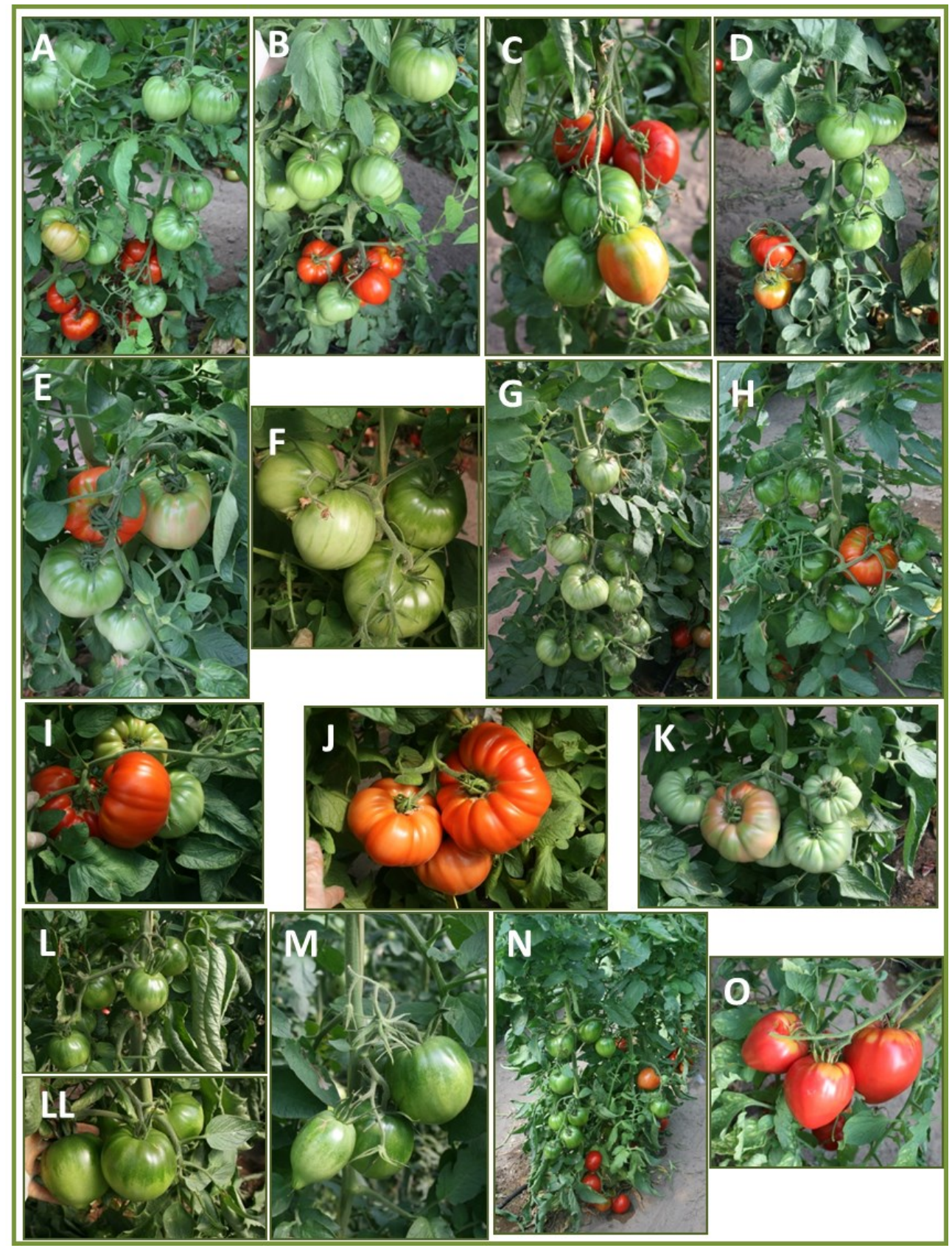

Figura 1.- Selección de entradas de tomate 'Valenciano' pertenecientes a los tipos "Femella" (A a H), "Xato" ((I a K), "Masclet" (L a N) y "Rosada" (O) 
Los aspectos de mayor interés de la colección de entradas de tomate 'Valenciano' conservadas en el banco de germoplasma del COMAV son la gran variabilidad que contiene para muchos caracteres de interés, al tratarse de entradas que no han sido utilizadas en programas de mejora, y la adaptación a distintas condiciones agroclimáticas, ya que proceden, según los datos presentados, de 23 comarcas de las tres provincias.

\section{AGRADECIMIENTOS}

Los trabajos descritos en esta publicación han sido financiados mediante sucesivos proyectos del INIA (Ministerio de Economía y Competitividad). El último de ellos es el de referencia RFP2015-00013-0000 . También han recibido financiación del programa de investigación e innovación Horizonte 2020 de la Unión Europea a través del contrato No 634561 (TRADITOM).

\section{REFERENCIAS BIBLIOGRÁFICAS}

Cebolla J. 2005. Recuperación de variedades tradicionales de tomate y pimiento. Caracterización y mejora genética. Tesis doctoral. Universitat Politècnica de València.

Cebolla-Cornejo J, Soler S, Nuez F. 2007. Genetic erosion of traditional varieties of vegetable crops in Europe: tomato cultivation in Valencia (Spain) as a case study. Intl J Plant Prod 1: 113-127.

Cortés-Olmos C, Valcárcel JV, Roselló J, Díez MJ, Cebolla-Cornejo J. 2015. Traditional Eastern Spanish varieties of tomato. Sci Agri 5: 420431.

Figás M, Prohens J, Raigón MD, Fernández de Córdiva P, Fita A, Soler S. 2015. Characterization of a collection of local varieties of tomato (Solanum lycopersicum L.) using conventional descriptors and the highthroughput phenomics tool Tomato Analyzer. Genet Resour Crop Evol 62: 189-204.

IPGRI. 1996. Descriptors for tomato (Lycopersicon spp.). International Plant Genetic Resources Institute, Rome, Italia.

Rao NK, Hanson J, Dulloo ME, Ghosh K, Novell D, Larinde M. 2006. Manual of seed handling in genebanks. Handbooks for Genebanks No. 8. Bioversity International, Rome, Italy. 
Soler S, Figás M, Díez MJ, Granell A, Prohens J. 2016. Tomate. E: Las variedades locales en la mejora genética de plantas. Ruiz de Galarreta JI y Prohens J (Eds). Servicio Central de Publicaciones del Gobierno Vasco. Vitoria-Gasteiz. 